Compati bi lity between hi gh energy particle conf i nement and magnet ohydrodynamic st abi I i ty in the i nward-shifted pl asmas of the Large Hel i cal Devi ce

\begin{tabular}{|l|l|}
\hline $\begin{array}{l}\text { j our nal or } \\
\text { publ i cat i on } \mathrm{titl} \text { e }\end{array}$ & Physi cs of Pl asmas \\
\hline vol une & 9 \\
\hline nunber & 5 \\
\hline page range & $2020-2026$ \\
\hline year & $2002-0423$ \\
\hline URL & ht t p: //hdl . handl e. net $/ 10655 / 1739$ \\
\hline
\end{tabular}




\title{
Compatibility between high energy particle confinement and magnetohydrodynamic stability in the inward-shifted plasmas of the Large Helical Device ${ }^{a)}$
}

\author{
O. Kaneko, ${ }^{\text {b) }}$ A. Komori, H. Yamada, N. Ohyabu, K. Kawahata, Y. Nakamura, K. Ida, \\ S. Murakami, T. Mutoh, S. Sakakibara, S. Masuzaki, N. Ashikawa, ${ }^{c)}$ M. Emoto, \\ H. Funaba, M. Goto, H. Idei, K. Ikeda, S. Inagaki, N. Inoue, M. Isobe, K. Khlopenkov, \\ S. Kubo, R. Kumazawa, T. Minami, J. Miyazawa, T. Morisaki, S. Morita, S. Muto, \\ Y. Nagayama, N. Nakajima, H. Nakanishi, K. Narihara, K. Nishimura, N. Noda, \\ T. Notake, ${ }^{\text {d) }}$ T. Kobuchi, ${ }^{c)}$ Y. Liang, S. Ohdachi, Y. Oka, M. Osakabe, T. Ozaki, \\ B. J. Peterson, A. Sagara, K. Saito, ${ }^{\text {d) }}$ R. Sakamoto, M. Sasao, K. Sato, M. Sato, \\ T. Seki, T. Shimozuma, M. Shoji, H. Suzuki, M. Takechi, Y. Takeiri, N. Tamura, ${ }^{c}{ }^{\text {' }}$ \\ K. Tanaka, K. Toi, T. Tokuzawa, Y. Torii, ${ }^{\text {d) }}$ K. Tsumori, I. Yamada, S. Yamamoto, ${ }^{d)}$ \\ M. Yokoyama, Y. Yoshimura, M. Yoshinuma, K. Y. Watanabe, T. Watari, Y. Xu, \\ K. Itoh, K. Matsuoka, K. Ohkubo, I. Ohtake, T. Satow, S. Sudo, K. Yamazaki, \\ Y. Hamada, O. Motojima, and M. Fujiwara \\ National Institute for Fusion Science, Oroshi-cho 322-6, Toki, Gifu 509-5292, Japan
}

(Received 29 October 2001; accepted 10 December 2001)

\begin{abstract}
The experimentally optimized magnetic field configuration of the Large Helical Device [A. Iiyoshi et al., Nucl. Fusion 39, 1245 (1999)], where the magnetic axis is shifted inward by $15 \mathrm{~cm}$ from the early theoretical prediction, reveals $50 \%$ better global energy confinement than the prediction of the scaling law. This configuration has been investigated further from the viewpoints of high energy particle confinement and magnetohydrodynamic (MHD) stability. The confinement of high energy ions is improved as expected. The minority heating of ion cyclotron range of frequency was successful and the heating efficiency was improved by the inward shift. The confinement of passing particles by neutral beam injection was also improved under low magnetic field strength, and there could be obtained an almost steady high beta discharge up to $3 \%$ in volume average. This was a surprising result because the observed pressure gradient exceeded the Mercier unstable limit. The observed MHD activities became as high as beta but they did not grow enough to deteriorate the confinement of high energy ions or the performance of the bulk plasma, which was still 50\% better than the scaling. According to these favorable results, better performance would be expected by increasing the heating power because the neoclassical transport can also be improved there. (C) 2002 American Institute of Physics. [DOI: 10.1063/1.1448345]
\end{abstract}

\section{INTRODUCTION}

The confinement of high energy particles and the high beta operation were critical design issues for helical fusion reactors. The recent development in the theories of designing modern stellerators, as well as magnetohydrodynamic (MHD) stability, have made it possible to design magnetic field configurations for optimizing these issues. However, in the real situation, the performance of helical plasmas is determined by anomalous nature, and the experimental efforts are focused on the optimization of plasma confinement at first, and then the designed concepts are verified at the optimized point, which may not always be the same as predicted.

The Large Helical Device (LHD) ${ }^{1}$ is a modern $l=2$ heliotron with a pitch number $m=10$, the design of the magnetic field configuration of which was optimized for high

\footnotetext{
${ }^{\text {a)}}$ Paper QI1 2, Bull. Am. Phys. Soc. 46, 246 (2001).

b) Invited speaker.

${ }^{c}$ Graduate University for Advanced Studies, Hayama 240-0193, Japan.

${ }^{d)}$ Department of Energy Engineering and Science, Nagoya University, Nagoya 464-8603, Japan.
}

energy particle confinement, stability and equilibrium of high beta plasma, and the formation of a clear helical divertor field structure. It is a superconducting machine of major $\left(R_{\mathrm{ax}}\right)$ and minor radius $(a)$ of $3.75 \mathrm{~m}$ and $0.6 \mathrm{~m}$ with maximum toroidal magnetic field strength of $2.75 \mathrm{~T}$ at the plasma center. The rotational transform is 0.35 at the center and 1.2 at the edge in the standard configuration of $R_{\mathrm{ax}}=3.75 \mathrm{~m}$. The LHD has the flexibility to change the plasma configuration by using a set of superconducting poloidal coils. Among these shaping parameters, the position of the magnetic axis is the most important to determine the performance of the plasma because the MHD stability is predicted to be improved when the axis is shifted outward where the magnetic well region increases, while the excursion of the particle orbit from the magnetic surface becomes small by inward shift, which reduces the neoclassical transport as well. The optimized position was determined to be $3.75 \mathrm{~m}$ in the design phase of the machine, which was $15 \mathrm{~cm}$ inward from the center of the helical winding coil $(3.9 \mathrm{~m})$.

Since the start of experiments in 1998, the performance of LHD plasma has been improved by increasing the heating 
power and the magnetic field strength. Through the experimental studies, we tried optimizing the position of the magnetic axis in terms of plasma energy confinement, and found that the $15 \mathrm{~cm}$ inner shift from the standard position $\left(R_{\mathrm{ax}}\right.$ $=3.6 \mathrm{~m}$ ) showed excellent performance, that is, 1.5 times better than the one predicted by the scaling law deduced from the medium size stellerators/heliotrons: the international stellerator scaling $1995^{2}$ (ISS-95). ${ }^{3}$ According to the design study of LHD, this position is predicted to be favorable for the high energy particle confinement, but the MHD stability of high beta plasma is another worrisome question. In this paper, we will describe that the compatibility between the high energy confinement and the MHD stability still holds good at this position.

\section{OPTIMIZATION OF MAGNETIC CONFIGURATION ON ENERGY CONFINEMENT}

The magnetic field configuration of LHD, such as the position of the magnetic axis or the shape of the plasma cross section, can be widely changed by using the poloidal shaping coils or by changing the current distribution in the helical coils. They are also related to the change of the profile of rotational transform or the divertor field structure, including the case of inner wall limiter discharge. A systematic study has been carried out recently to find the best magnetic configuration for the plasma energy confinement of neutral beam injection (NBI) heated plasma. ${ }^{4}$ In the study, the performances of plasma were compared by changing the position of the magnetic axis $\left(3.55 \mathrm{~m} \leqslant R_{\mathrm{ax}} \leqslant 4.0 \mathrm{~m}\right)$ and then the averaged shape of the poloidal cross section $(0.92 \leqslant \kappa \leqslant 1.17$ where $\kappa$ is averaged ellipticity) at $3.6 \mathrm{~m}$. The results on the global energy confinement time are summarized in Fig. 1. Because the minor radius also varies as the magnetic axis varies, the plasma performance is compared by the improvement factor of energy confinement time from the empirical scaling ISS-95 $\left[\tau_{E}=0.08 a^{2.21} R^{0.65} P^{-0.59} n_{e}{ }^{-0.51} B^{0.80}\right.$ $\left.(\iota / 2 \pi)_{2 / 3}{ }^{0.40}\right]$. The dependence of other parameters in ISS-95, such as input power $(P)$, electron density $\left(n_{e},\right)$ and toroidal field strength (B), agree well with the experimental results in LHD in the case of $R_{\mathrm{ax}} \leqslant 3.6 \mathrm{~m}$ and in the collisional regime of $R_{\mathrm{ax}} \leqslant 3.75 \mathrm{~m}$, which is weakly gyro-Bohm. Then Fig. 1 indicates that the coefficient of the scaling strongly depends on the magnetic axis, and not very much on averaged $\kappa$ although its dependence was studied only at $R_{\mathrm{ax}}=3.6 \mathrm{~m}$. A similar effect of the magnetic axis was also observed in medium-sized heliotrons where the plasma is in the plateau regime. ${ }^{5}$

One of the specific features of LHD plasma is the existence of a natural pedestal in the electron temperature profile, ${ }^{6}$ which improves the global energy confinement of LHD plasmas compared with those of medium-sized heliotrons. ${ }^{7}$ In this case, the confinement of the core part [subtracting the base of pedestal part; $\int I\left(W_{p}(\rho)\right.$ $\left.\left.-W_{p}(0.9)\right) d \rho\right]$ does not change. However, the shift of the magnetic axis also improves the confinement of the core part. The best position of the magnetic axis is around $3.55 \mathrm{~m}$, from Fig. 1. It is interesting that the prospected neoclassical transport in the collisionless regime also reduces as the mag- [vacuum magnetic field configuration]

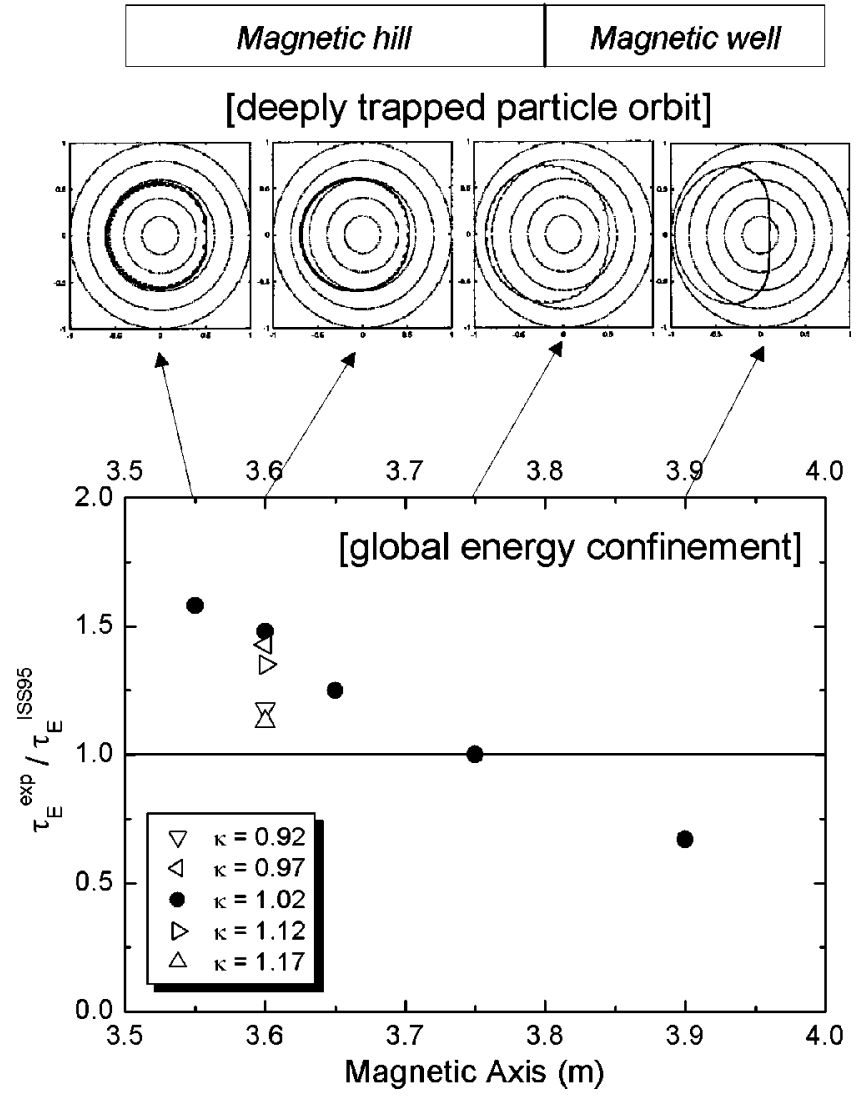

FIG. 1. Experimentally observed dependence of global energy confinement on the various magnetic field configurations in the LHD (position of magnetic axis $R_{\mathrm{ax}}$, and averaged plasma ellipticity $\kappa$ ). The predicted changes of a deeply trapped particle orbit in Boozer coordinate are shown as $R_{\mathrm{ax}}$. The position where the magnetic well appears in the vacuum magnetic field configurations is also indicated.

netic axis shifts inward, and it takes the minimum value at $R_{\text {ax }}=3.53 \mathrm{~m}^{8}{ }^{8}$ This dependence is stronger than that of experimentally observed anomalous transport. Both transports become comparable under some conditions. Because the anomalous transport does not strongly depend on the collisionality (weakly gyro-Bohm), the neoclassical transport may surpass the anomalous one in the collisionless regime when the magnetic axis is shifted outward. Actually, the deterioration of confinement occurred as the collisionality decreased at $R_{\mathrm{ax}} \leqslant 3.75 \mathrm{~m}$. ${ }^{4}$ This situation is useful for studying the neoclassical characteristics in the LHD. One of the examples was the transition of transport nature from ion root to electron root in the NBI discharge, and the effect of radial electric field on the transport was clearly observed. ${ }^{9}$

As yet, this kind of deterioration was not observed at $R_{\text {ax }}=3.6 \mathrm{~m}$, even when the collisionality $\nu_{b}^{*}$ decreased as low as 0.05 , where $\nu_{b}^{*}=\nu_{\mathrm{ei}} q R / \varepsilon_{t} 1.5 / v^{e}{ }_{\text {th }}$. Further improvement would be possible if we could suppress the anomalous transport in the reactor relevant regime of $\nu_{b}^{*} \sim 10^{-2}$. Therefore, this configuration is promising as a new standard of LHD, if other conditions are satisfied.

\section{CONFINEMENT OF HIGH ENERGY IONS}

The vacuum magnetic field configuration of LHD was designed to have a clear divertor field structure by positive 


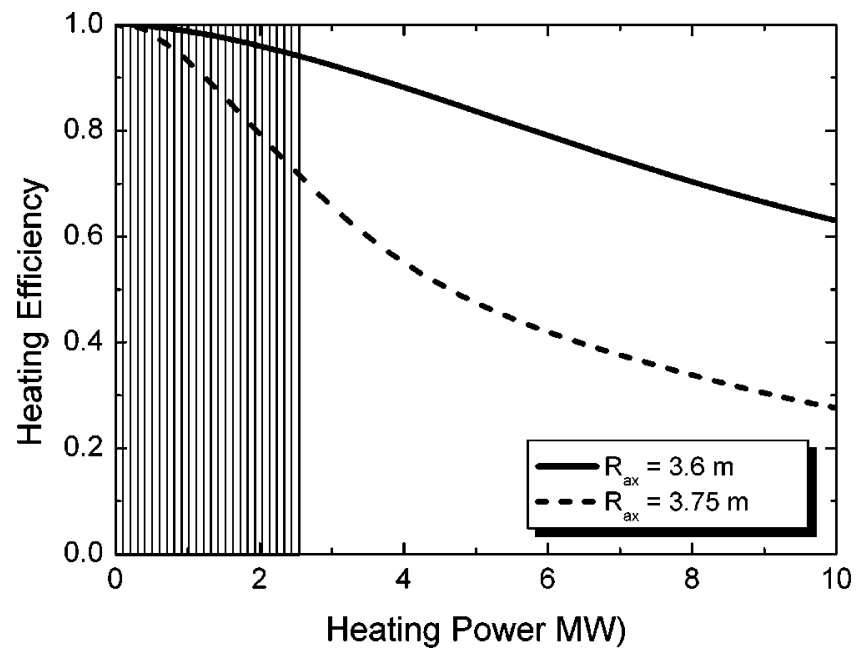

FIG. 2. Heating efficiency of ICRF minority heating. The averaged electron density is $2 \times 10^{19} \mathrm{~m}^{-3}$. Solid line shows the case of $R_{\mathrm{ax}}=3.6 \mathrm{~m}$ and dashed line $R_{\mathrm{ax}}=3.75 \mathrm{~m}$. Hatched region indicates the current power level of ICRF.

pitch modulation of the helical coil, and the improvement of trapped particle orbit was guaranteed by the inward shift of the magnetic axis from the center of helical coil. From the viewpoint of good particle orbit, the best position is predicted to be $R_{\mathrm{ax}}=3.53 \mathrm{~m}$, and a more inward shift of the magnetic axis from the standard position $(3.75 \mathrm{~m})$ is expected to improve the confinement of high energy ions.

Experimentally, the confinement of high energy ions can be investigated by using those produced by plasma heating devices because deuterium is not available for a working gas. Although the behavior of helically trapped ions is interesting from this point of view, the main heating method was a tangentially injected neutral beam to avoid producing high energy trapped ions. ${ }^{10}$ The ion cyclotron range of frequency (ICRF) heating was prepared for the purpose of target plasma production of NBI using ion Bernstein wave heating. ${ }^{11}$ However, through the experiments, the minority heating has been found to be very efficient in the inward shifted discharges. ${ }^{12}$

\section{A. Confinement of perpendicularly accelerated ions (ICRF heating)}

ICRF heating of helical plasma has been carried out in some devices, ${ }^{13-15}$ and the plasma production and heating was demonstrated using the electron heating scheme. However, ion heating using a minority heating scheme has not been very successful in the medium-sized helical systems, which is considered to be attributed to the existence of a large loss cone for perpendicularly accelerated ions. In the LHD, due to its large minor radius, the slowing down process can compete the loss of helically trapped minority ions, which are accelerated perpendicularly by the ICRF wave. Therefore, the improvement of fast ion orbit can affect the heating efficiency of ICRF in minority heating, and in this sense, the inward shift is preferable for ICRF heating. Figure 2 shows a simulation of energy absorption of an accelerated minority ion by ICRF, ${ }^{16}$ where the ion is accelerated at the resonant point when it passes and loses energy by friction
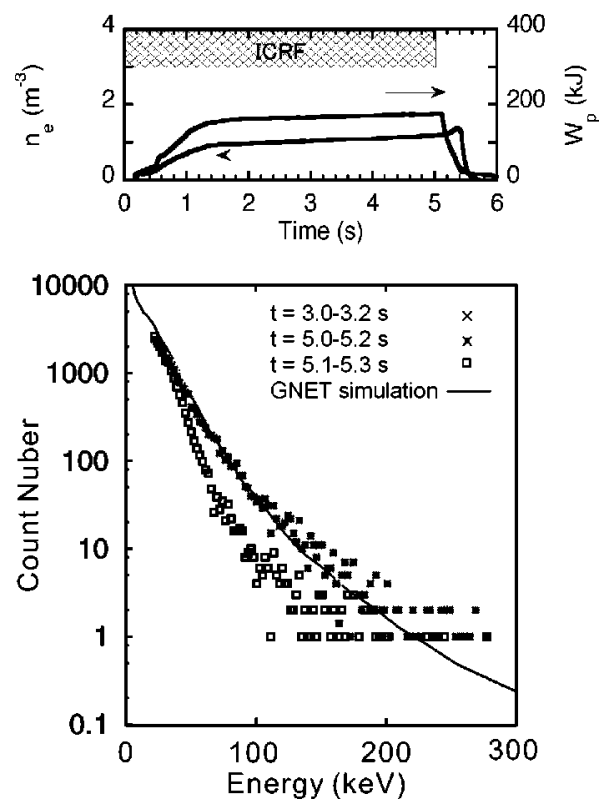

FIG. 3. Energy spectrum of charge exchanged neutrals of minority hydrogen ions during ICRF heating measured by a natural diamond detector. Solid line in the figure is a predicted spectrum of neutral particles via charge exchange based on the energy distribution of minority ions calculated by GNET code.

through the excursion until it runs away from the confinement zone (inside the last closed magnetic surface). The efficiency becomes low when the slowing down time becomes long, that is, the bulk temperature becomes high or the density is low, and therefore the efficiency becomes worse for high power heating. The figure shows clear improvement of heating efficiency when the magnetic axis is shifted from $3.75 \mathrm{~m}$ to $3.6 \mathrm{~m}$, and good minority heating was expected at $R_{\mathrm{ax}}=3.6 \mathrm{~m}$.

The ICRF heating was performed in helium plasma with hydrogen as the minority ion. It could additionally heat NBI plasmas or sustain a plasma by itself without any increment of density or radiation power. ${ }^{12}$ The antennas can move radially and cope with the magnetic axis shift. In the inward shifted discharges, the heating efficiency was similar to that of NBI. The growth of a high energy tail up to $300 \mathrm{keV}$ was observed in hydrogen energy during ICRF heating. ${ }^{17}$ Figure 3 shows a perpendicular energy spectrum of charge exchanged neutral hydrogen which was observed by the natural diamond detector. ${ }^{18}$ The obtained energy spectrum agrees with the result of 5D simulation code GNET ${ }^{19}$ in which the energy and spatial distribution of minority ions are calculated. Figure 4 compares the increment of plasma-stored energy at $R_{\text {ax }}=3.6 \mathrm{~m}$ with that at $3.75 \mathrm{~m}$ when the ICRF was applied to the plasma produced by $1 \mathrm{MW}$ NBI. It was found that the increment of stored energy of $3.6 \mathrm{~m}$ was more than twice as large as that of $3.75 \mathrm{~m}$. As shown in Fig.1, the bulk energy confinement of $3.6 \mathrm{~m}$ is 1.5 times better than that of $3.75 \mathrm{~m}$. The obtained increment of stored energy agrees well with the prediction from ISS-95 at $3.6 \mathrm{~m}$, but the efficiency of $3.75 \mathrm{~m}$ is worse than the prediction, as shown in the figure. This low efficiency qualitatively agrees with the result shown in Fig. 2 where about $70 \%$ of reduction from the 


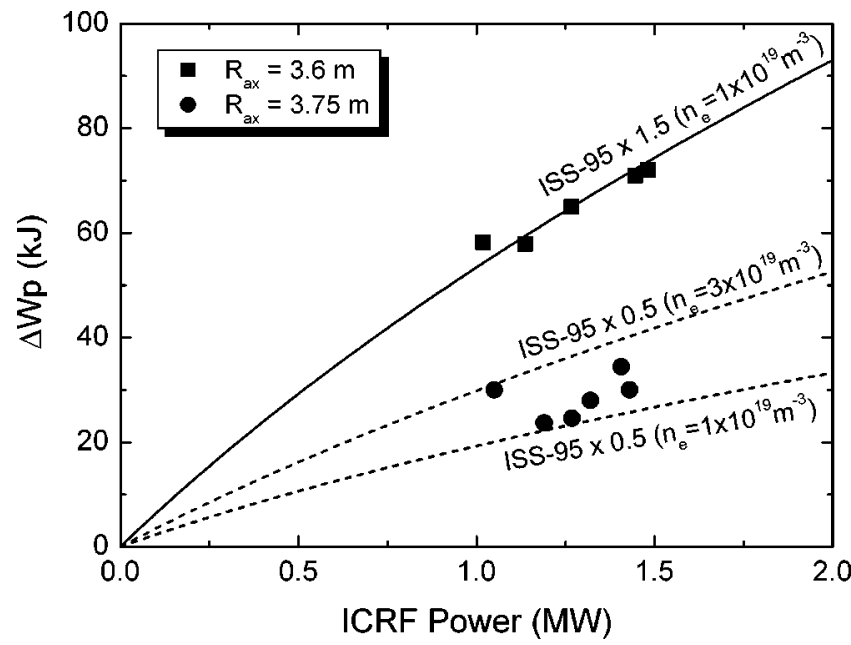

FIG. 4. The increments of stored energy by ICRF heating of NBI plasma are compared between $R_{\mathrm{ax}}=3.6 \mathrm{~m}$ and $3.75 \mathrm{~m}$ with the ISS-95 scaling. The magnetic field strength is $2.75 \mathrm{~T}$ and the target plasma density is around 1 $\times 10^{19} \mathrm{~m}^{-3}$ at $R_{\mathrm{ax}}=3.6 \mathrm{~m}$ and $1-3 \times 10^{19} \mathrm{~m}^{-3}$ at $R_{\mathrm{ax}}=3.75 \mathrm{~m}$. The absorbed power of NBI is $1 \mathrm{MW}$.

ISS-95 is anticipated when the power of NBI is included.

The energy spectrum shown in Fig. 3 was measured at $R_{\mathrm{ax}}=3.6 \mathrm{~m}$. The experimentally observed difference in the perpendicular high energy tail was, however, small between $R_{\mathrm{ax}}=3.75 \mathrm{~m}$ and $3.6 \mathrm{~m}$. This fact does not contradict the difference of orbits between $3.6 \mathrm{~m}$ and $3.75 \mathrm{~m}$. Because the excursion of perpendicularly accelerated ions is larger at $R_{\mathrm{ax}}=3.75 \mathrm{~m}$, they pass the plasma periphery where the neutral density is high. According to the simulation by GNET, the flux of high energy ions becomes even slightly larger in the energy spectrum of $3.75 \mathrm{~m}$, which is consistent with the experimental results. ${ }^{20}$

\section{B. Confinement of high energy passing ions (NBI heating)}

The confinement of passing particles is predicted to be good in the LHD so that all the neutral beams are injected tangentially. Two beam lines aim in the opposite direction to cancel the beam driven current. Because of a large major radius, the hydrogen beam energy should be as high as 180 $\mathrm{keV}$ for heating the center of plasma. The negative-ion-based technology was introduced to realize such high energy beams, which results in no partial energy components existing in the beam. Therefore, the confinement of fast ions is also a concern, especially under low magnetic field strength for high beta study. Experimentally, the heating efficiency under high field is good, and no difference was observed between co-injection and counter injection. No difference was observed either when the magnetic field strength went down to $0.75 \mathrm{~T}$, where the toroidal drift of the particle orbit from the magnetic surface can be nearly a third of minor radius $\left(\sqrt{\varepsilon_{t}} \rho_{p} / a \sim 0.3\right)$. Figure 5 shows a dependence of plasma-stored energy on the magnetic field strength keeping the input power and the target density constant. The figure shows good agreement of toroidal field dependence with the scaling ISS-95, which indicates that the heating efficiency

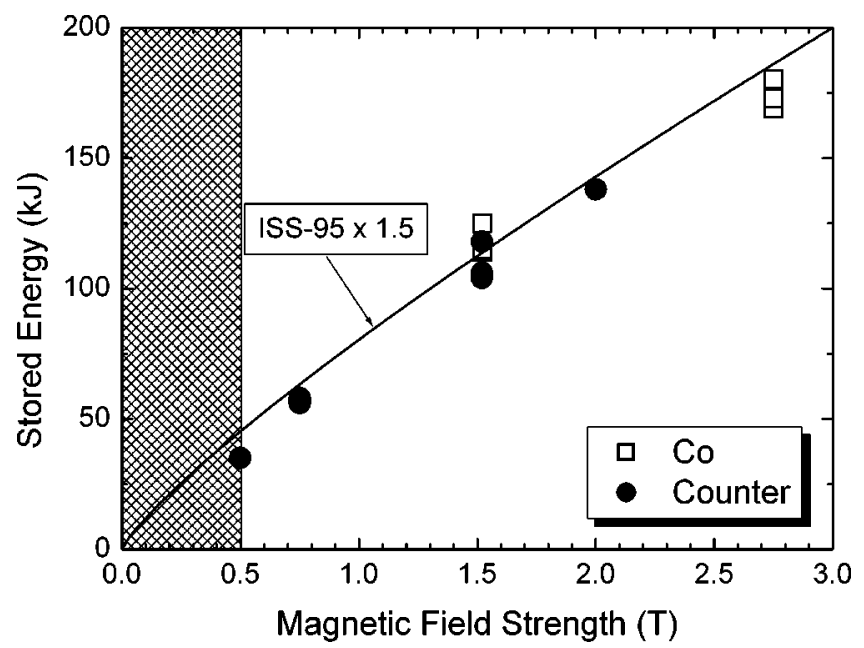

FIG. 5. Dependence of neutral beam heating efficiency on the magnetic field strength. Injection power and the target plasma density are $1.7 \mathrm{MW}$ and 1 $\times 10^{19} \mathrm{~m}^{-3}$, respectively. Hatched region indicates where the interference with ICRF antenna occurs. Solid line shows the predicted stored energy based on the ISS-95 scaling.

does not deteriorate as low as $0.75 \mathrm{~T}$. At $0.4 \mathrm{~T}$ the interference was observed with the side limiter of the ICRF antenna, which is located about $12 \mathrm{~cm}$ apart from the last closed magnetic surface, in the discharge at $R_{\mathrm{ax}}=3.6 \mathrm{~m}$. It was also observed at $0.5 \mathrm{~T}$ in the case at $R_{\mathrm{ax}}=3.75 \mathrm{~m}$. These are clear observations that the high energy ions deviate far from magnetic flux surfaces.

A problem in the operation under low magnetic field strength in helical systems was plasma initiation. We have developed a new technique to initiate plasma by NBI alone in the LHD. ${ }^{21}$ In this method, NBI is injected at first into the vacuum vessel with prefilled neutral gas. Then a small part of the beam is ionized by collision with background neutral gas and trapped in the vacuum magnetic field (LHD is a superconducting machine and the magnetic field is always present). These trapped fast ions become the energy source of the following discharge. Therefore, the confinement of fast ions determines the success of startup. It takes time to build up plasma from the start of the beam, and this "waiting time" becomes long as the beam current decreases. Figure 6 shows the time evolutions of plasma density buildup by counter beam injection under the various magnetic field strengths. In this series of experiments no conditions were changed besides magnetic field strength, and the waiting time becomes long under $0.75 \mathrm{~T}$. Because the waiting times are the same between $2.75 \mathrm{~T}$ and $1.52 \mathrm{~T}$, this delay is thought to be the effect of increasing direct orbit loss of the beam. The method is very reliable and high beta studies have been pushed forward.

\section{HIGH BETA DISCHARGE AND MHD CHARACTERISTICS}

The magnetic axis of $3.6 \mathrm{~m}$ was not chosen in the design phase of LHD because it becomes a magnetic hill configuration and the plasma was considered to be MHD unstable at high beta. In order to check this situation, we have tried producing high beta plasmas at $R_{\mathrm{ax}}=3.6 \mathrm{~m}$, and studied their 


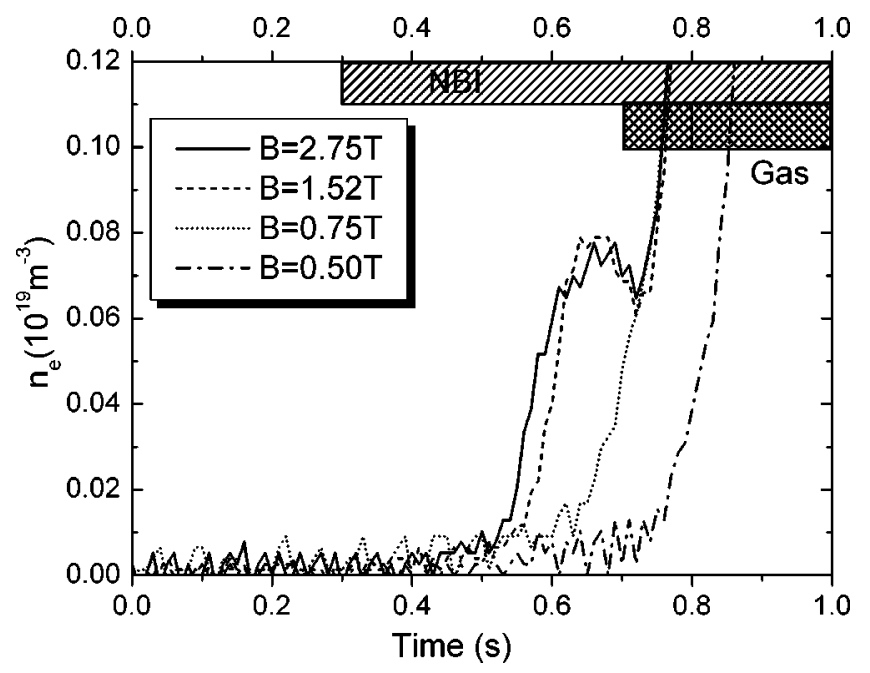

FIG. 6. Time evolution of plasma initiation by neutral beam under different magnetic field strength. The beam power is $1.7 \mathrm{MW}$. The start time of gas puffing is delayed by $0.1 \mathrm{~s}$ at $B_{T}=0.5 \mathrm{~T}$ from other cases due to a slow density buildup.

MHD characteristics carefully. Because the available heating power is limited, the discharges had to be performed under the magnetic field strength as low as possible. As described in the preceding section, under such a low field the only tool for plasma production and heating is NBI. By considering the dependence of beta, global energy confinement, and heating efficiency on the magnetic field strength, high beta experiments were carried out at $0.5 \mathrm{~T}$.

Figure 7 shows time evolutions of plasma parameters

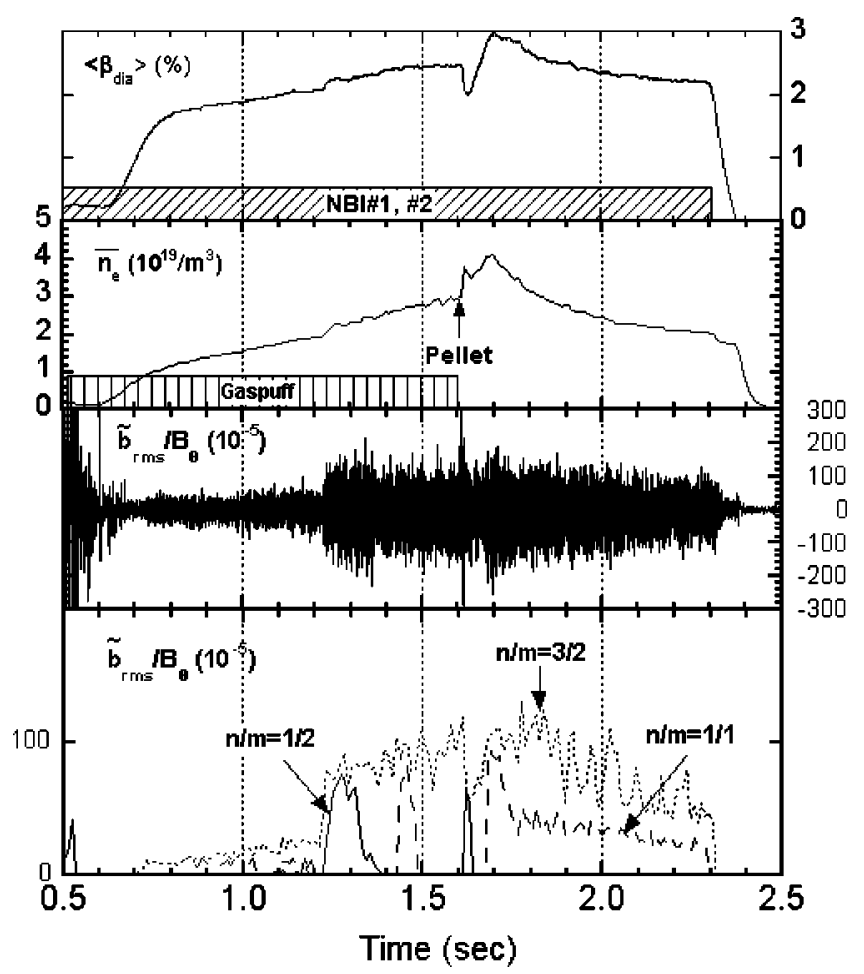

FIG. 7. Time evolution of averaged beta, electron density, magnetic fluctuation, and amplitude of coherent modes in a high beta discharge at $B_{t}$ $=0.5 \mathrm{~T}$.

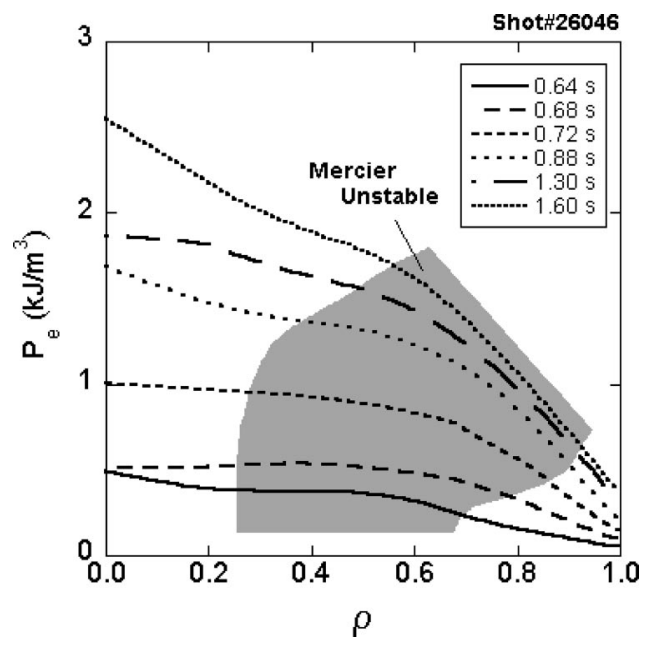

FIG. 8. Time evolution of electron pressure profiles in the discharge of Fig. 7. A predicted Mercier unstable region is also shown.

and magnetic fluctuations during a discharge when the volume averaged beta value $\left\langle\beta_{\text {dia }}\right\rangle$ of $3 \%$ was achieved, which is, so far, the highest value achieved in the LHD. Here, $\left\langle\beta_{\mathrm{dia}}\right\rangle$ is defined using the diamagnetically measured stored energy and the vacuum magnetic pressure. In this discharge, the gas puff was continued until $1.6 \mathrm{~s}$, and then a pellet was injected. The $\left\langle\beta_{\mathrm{dia}}\right\rangle$ increased up to $2.5 \%$ during gas puffing as density increased, and the highest beta was obtained at the density growing phase after pellet injection. The level of magnetic fluctuation increased with beta and enlarged at $1.23 \mathrm{~s}$ due to the appearance of low- $n$ coherent modes, such as $n / m=1 / 2,1 / 1$, and $3 / 2$, as shown in the figure. Among them the $1 / 2$ mode disappeared as $\left\langle\beta_{\mathrm{dia}}\right\rangle$ increased more than $2.3 \%$.

Figure 8 shows the time evolution of electron pressure profiles of this discharge. The ion temperature has almost the same value as the electron in this density range in LHD. As shown in the figure, a steep pressure gradient exists at the peripheral regions. It is noted that the obtained pressure gradient $(|d \beta / d \rho|)$ in the hatched region in the figure exceeds Mercier stability limits. At $\rho=0.9$, where a rational surface of $1 / 1$ exists, it becomes Mercier unstable over $1.8 \%(t$ $>0.33 \mathrm{~s})$, and it is predicted to be unstable to low- $n$ mode when $\left\langle\beta_{\mathrm{dia}}\right\rangle>2.1 \%(t>1.3 \mathrm{~s})$ theoretically. Although the coherent modes seem to appear when the pressure gradient is close to the low- $n$ unstable limit, the coincidence with the theory is not very good. On the other hand, at $\rho=0.5$ where the rational surface of $2 / 1$ exists, the obtained pressure gradient is Mercier unstable below $2 \%(t<1.4 \mathrm{~s})$, and it is predicted to enter the second stability region when the beta continues to increase. The disappearance of $2 / 1$ coherent mode corresponds to this timing $(t=1.4 \mathrm{~s})$, but its onset is not clearly explained. So far, the pressure gradients at a peripheral region increased almost linearly as beta increased. ${ }^{22}$

A remarkable result is that the plasma was stable even if it was predicted to be Mercier unstable. Although small activities were observed in Soft X or electron cyclotron emissions (ECE) measurements in some cases, we have not yet experienced any plasma soft disruption or confinement deg- 


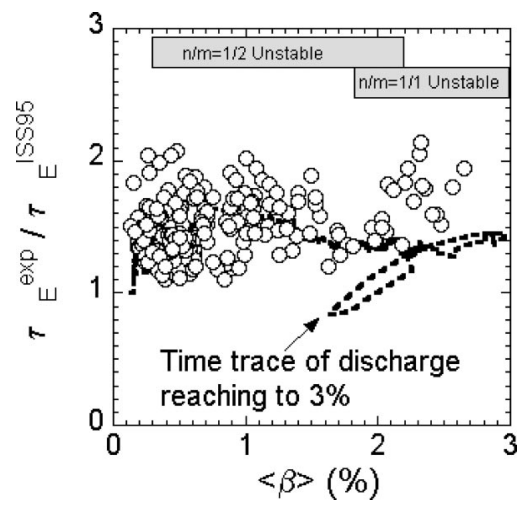

FIG. 9. Dependence of the improvement of global energy confinement on volume-averaged beta. No degradation was found up to $3 \%$. Time trace of the discharge of Fig. 7 is also shown with the predicted unstable conditions.

radation due to instability accompanied by the high beta value. Actually, the improved characteristics of global energy confinement do not depend on beta until 3\%, as shown in Fig. 9, where the time trace of the discharge shown in Fig. 7 is also indicated with the predicted unstable conditions.

\section{SUMMARY AND DISCUSSIONS}

It was experimentally confirmed that the compatibility between high energy particle confinement and high beta achievement holds good in the inward-shifted configuration of LHD. The anomalous thermal diffusion is suppressed best at $R_{\mathrm{ax}}=3.55 \mathrm{~m}$, as seen from Fig. 1. This magnetic configurational effect indicates that there is other parameter(s) to be considered in the conventional scaling law. However, through the change of magnetic configurations, the magnetic shear does not vary enough to explain the experimental result. The fact that the confinement is independent of beta suggests that the magnetic well configuration does not affect the confinement. A difference can be seen in the structure of multihelicity, which is related to the improvement of particle orbit, but so far we have no idea that it has anything to do with suppression of turbulence.

The neoclassical transport is improved by the inward shift as the theory predicts. Good confinement of high energy ions was experimentally confirmed. The fact that the improvement of confinement by magnetic axis shift has the same tendency in both anomalous and neoclassical nature is one of the important experimental results from the optimization of magnetic configurations in the LHD. Although the best position is expected at around $R_{\mathrm{ax}}=3.55 \mathrm{~m}$ both anomalously and neoclassically, the tangency radius of NBI is set as $3.7 \mathrm{~m}$, which was determined by optimizing an absorbed profile of beams in the plasma of standard magnetic axis of $3.75 \mathrm{~m}$, and the beam deposition becomes worse for such a large inward shift. Changing the sight line of the beam more inwardly will cause interference with the inner wall of vacuum vessel. Therefore, $3.6 \mathrm{~m}$ is set as our new standard axis to study the reactor relevant plasma.

Other heating tools can cope with the inward shift. Recently, the mirror driving system of electron cyclotron heating $(\mathrm{ECH})$ has been modified (it was also optimized based on

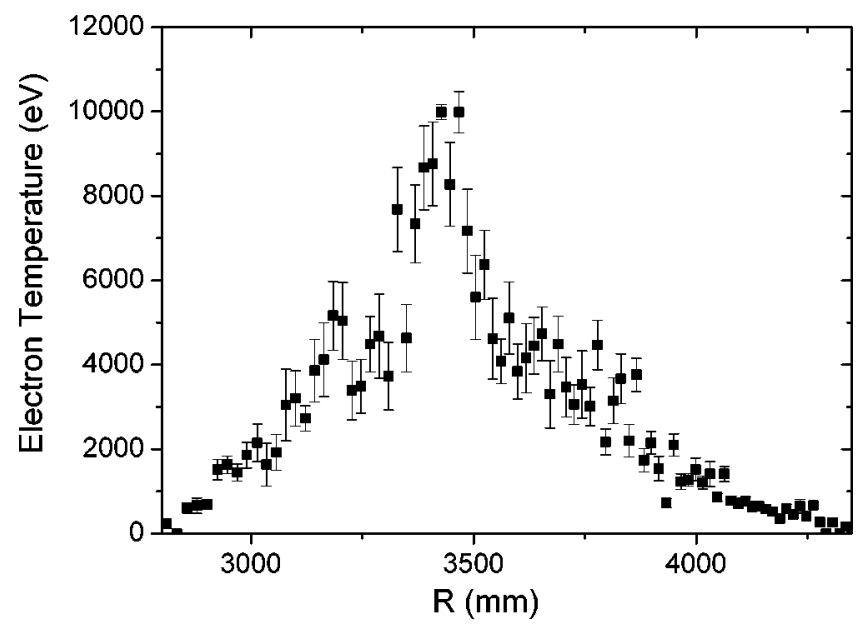

FIG. 10. Electron temperature profile obtained by central heating of ECH. The ECH power of $1 \mathrm{MW}$ (combination of fundamental and second harmonic heating) was focused on the magnetic axis within the region $\rho$ $\sim 0.1$. The electron density had a flat profile and was about 0.6 $\times 10^{19} \mathrm{~m}^{-3}$.

the standard axis of $3.75 \mathrm{~m}$ before), and the rays of $\mathrm{ECH}$ can be focused in the center of the plasma even at $R_{\mathrm{ax}}=3.5 \mathrm{~m}$, where the resonance layer can coincide with the magnetic axis. Figure 10 shows a steep electron temperature profile obtained by this localized heating. High electron temperature of around $9 \mathrm{keV}$ was obtained at the center, and the neoclassical feature is considered to be dominated in the central region where a strong radial electric field is expected to reduce transport. This is an important subject of the helical system, and vigorous studies are proceeding now.

Good MHD stability, in spite of the magnetic hill structure, is an unexpected experimental result of the inward shift discharges. It is suggested that even when the pressure gradient exceeds the Mercier limit a little, the growth rate stays small and the plasma is stable in the real situation. ${ }^{23}$ However, the obtained pressure profile far exceeds the limit. One of the possibilities for stabilizing plasma is the local flattening of the pressure profile. Unfortunately, we have to evaluate the density profiles using 12-chord far infrared (FIR) interferometer. Therefore, the spatial resolution is not good enough to evaluate the pressure profile in detail. More detailed measurement will be possible by Thomson scattering although it is not available to measure density due to insufficient calibration. For the other possibilities of stabilizing effect, such as plasma current or flow, we do not have any supporting experimental data so far. Recently, we have installed another neutral beam line, and the injected beam becomes unbalanced, so that we hope to get more information on the effect of plasma current or flow on MHD stability. More detailed theoretical analysis is also needed, including nonlinear treatment.

The experimental results show that the inward shift discharge revealed a good confinement and stability as well. Then there is the other important issue that should be clarified, that is, the helical divertor. One of the advantages of the $l=2$ heliotron is its clear divertor field structure. This feature still holds when the magnetic axis is shifted inward. Al- 


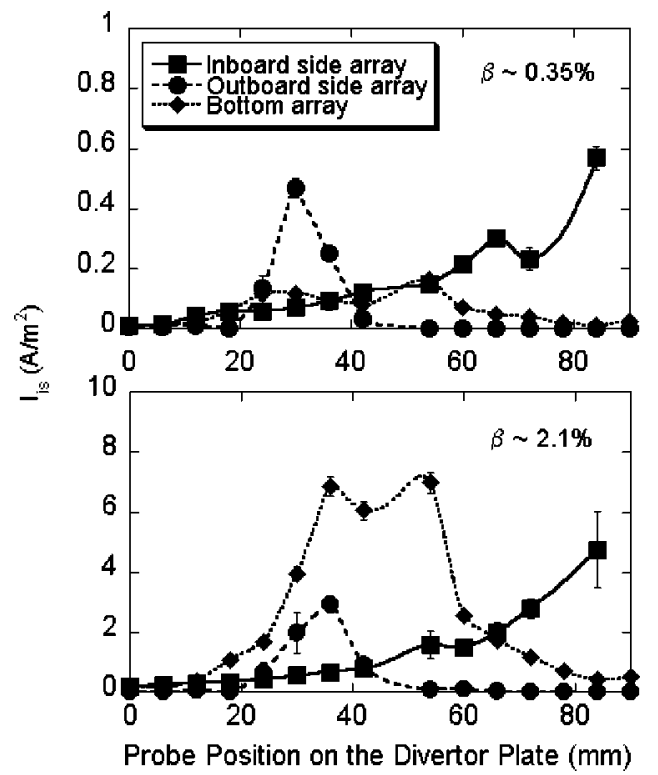

FIG. 11. Change of heat flux distribution on the divertor plates measured by Langmuir probe array when the beta increases. The increment of the bottom plate is larger than the other outboard and inboard plates.

though the structure of the divertor field lines begins to have fine structure and to spread over the divertor plates with the inward shift, it remains within a certain extent. This structure becomes more complicated when the plasma beta becomes high, but it is still bounded. Figure 11 shows the change of heat flux distribution as beta changes, which was measured by Langmuir probe array on the divertor plates at $R_{\mathrm{ax}}$ $=3.6 \mathrm{~m} .{ }^{24}$ It can be seen in the figure that the heat flux on the bottom plate increased much more strongly than other plates as beta increased. This is due to the Shafranov (outward) shift of plasma at high beta because the particles begin to escape from the separatrix of the outboard side when the plasma is shifted outward, and these particles go to the bottom plate. This tendency and the measured heat flux distribution on the divetor plate agree well with the calculation. Therefore, we think it possible to study the helical divertor at $R_{\text {ax }}=3.6 \mathrm{~m}$.

In conclusion, $R_{\mathrm{ax}}=3.6 \mathrm{~m}$ is a new standard configuration where the study of reactor relevant plasma can be pos- sible in LHD, which would extend the potential of the $l=2$ heliotron as a reactor.

\section{ACKNOWLEDGMENT}

The authors would like to acknowledge the members of the device engineering group for their operational support.

${ }^{1}$ A. Iiyoshi, A. Komori, A. Ejiri et al., Nucl. Fusion 39, 1245 (1999).

${ }^{2}$ U. Stroth, M. Murakami, R. A. Dory et al., Nucl. Fusion 36, 1063 (1996).

${ }^{3}$ H. Yamada, K. Y. Watanabe, K. Yamazaki et al., Nucl. Fusion 41, 901 (2001).

${ }^{4}$ H. Yamada, A. Komori, N. Ohyabu et al., Plasma Phys. Controlled Fusion 43, A55 (2001).

${ }^{5}$ O. Kaneko, S. Kubo, K. Nishimura et al., Plasma Physics and Controlled Nuclear Fusion Research, Washington, 1991 (International Atomic Energy Agency, Vienna, 1991), Vol. 2, p. 473.

${ }^{6}$ N. Ohyabu, K. Narihara, H. Funaba et al., Phys. Rev. Lett. 84, 103 (2000).

${ }^{7}$ H. Yamada, K. Y. Watanabe, S. Sakakibara et al., Phys. Rev. Lett. 84, $1216(2000)$.

${ }^{8}$ S. Murakami, A. Wakasa, H. Maassberg et al. (unpublished).

${ }^{9}$ K. Ida, H. Funaba, S. Kado et al., Phys. Rev. Lett. 23, 5297 (2001).

${ }^{10}$ O. Kaneko, Y. Takeiri, K. Tsumori et al., in Fusion Energy 1996, Montreal, 1996 (International Atomic Energy Agency, Vienna, 1997), Vol. 3, p. 539.

${ }^{11}$ K. Nishimura, R. Kumazawa, T. Mutoh et al., Fusion Eng. Des. 26, 179 (1995).

${ }^{12}$ T. Watari, T. Mutoh, R. Kumazawa et al., Nucl. Fusion 41, 325 (2001).

${ }^{13}$ T. Mutoh, H. Okada, O. Motojima et al., Nucl. Fusion 24, 1003 (1984).

${ }^{14}$ M. Kwon, T. D. Shepard, R. H. Goulding et al., Nucl. Fusion 32, 1225 (1992).

${ }^{15}$ D. A. Hartmann and G. Cattanei, in Fusion Energy 1998, Yokohama, 1998 (International Atomic Energy Agency, Vienna, 1999), Vol. 2, p. 575.

${ }^{16} \mathrm{~S}$. Murakami, N. Nakajima, M. Okamoto, and J. Nhuerenberg, Nucl. Fusion 39, 1165 (1999).

${ }^{17}$ M. Sasao, S. Murakami, M. Isobe et al., "Study of energetic ion transport in the Large Helical Device," Proceedings of the 18th International Atomic Energy Agency Fusion Energy Conference, Sorento, 2000 (International Atomic Energy Agency, Vienna, in press).

${ }^{18}$ M. Isobe, M. Sasao, S. Iiduka et al., Rev. Sci. Instrum. 72, 611 (2001).

${ }^{19}$ S. Murakami, U. Gasparino, H. Idei et al., Nucl. Fusion 40, 693 (2000).

${ }^{20}$ S. Murakami, H. Yamada, M. Isobe et al., in Proceedings of the 28th European Physical Society Conference on Controlled Fusion and Plasma Physics, Maderia, 2001 (European Physical Society, Petit-Lancy, in press), p. 4.045 .

${ }^{21}$ O. Kaneko Y. Takeiri, K. Tsumori et al., Nucl. Fusion 39, 1087 (1999).

${ }^{22}$ S. Sakakibara, H. Yamada, K. Watanabe et al., Nucl. Fusion 41, 1177 (2001).

${ }^{23}$ Y. Nakamura, K. Ichiguchi, M. Wakatani, and J. L. Johnson, J. Phys. Soc. Jpn. 58, 3157 (1989).

${ }^{24}$ S. Masuzaki, T. Morisaki, and N. Ohyabu (unpublished). 